\title{
15 Key Molecular and Metabolic Processes Used for Genetic Engineering to Improve Freezing Tolerance in Cereals
}

\author{
Alexandra Soltész, ${ }^{1 *}$ Wendy Harwood, ${ }^{2}$ Balázs Kalapos, ${ }^{1}$ Attila Vágújfalvi ${ }^{1}$ and \\ Gábor Galiba ${ }^{1}$ \\ ${ }^{1}$ Agricultural Institute, Hungarian Academy of Sciences, Martonvásár, Hungary; \\ ${ }^{2}$ ohn Innes Centre, Norwich, UK
}

\section{Introduction}

It has been estimated recently that cereals are harvested on 700 million hectares (Mha) worldwide (Dunwell, 2014), and also that, due to low temperature damage, worldwide losses in crop production amount to about US\$2 billion each year (Sanghera et al., 2011). In spite of the urgent need for more cold- or frost-tolerant cereal varieties, classical breeding programmes have shown limited progress in improving freezing tolerance (Thomashow, 1999). This lack of success is due mainly to the fact that the physiological process, i.e. the cold acclimation that leads to the development of freezing tolerance, is quite a complex quantitative trait. However, the deeper insight provided by different 'omics' technologies has made possibleknowledge-based engineering of more stress-resistant plants; while the recent developments in cereal transformation methodology offer the technology to realize these aims. Since many recently published book chapters and reviews summarize our current knowledge on plant abiotic stress tolerance, this chapter focuses particularly on freezing tolerance, especially in cereals.

\section{Changes in the Initial Phase of Cold Acclimation}

Decreasing temperature, day length and the alteration in light spectra during the autumn period are the three main environmental factors that provide signals for temperate zone perennial plants to prepare for upcoming frosty conditions (Badawi et al., 2007; Franklin, 2009; Sandre et al., 2011). In the cold acclimation process, however, only the contribution of cold temperature and day length are well elaborated, while the effect of light quality has not been researched extensively (Franklin and Whitelam, 2007). On sensing the changing environmental conditions, most of the metabolic processes are reprogrammed in a time-dependent manner. The first phase is associated with a decrease of hydraulic conductivity of roots, resulting in decreased water potential in leaves. To maintain the appropriate homeostasis of tissues, water status needs to be stabilized by

*E-mail: soltesz.alexandra@agrar.mta.hu 
stomata closure, which is regulated by abscisic acid (ABA). It is well documented that ABA content increases transiently in the early stage of cold stress response (Galiba et al., 1993). An increased level of ABA was found to coincide with the downregulation of other stress hormones, salicylic acid and jasmonic acid during an early phase of wheat response to cold stress (Kosová et al., 2012). The interaction among plant hormones is reviewed elsewhere (Galiba et al., 2013).

\section{CBF transcription factors}

After exposure to low temperature, in parallel with the enhanced ABA level, the transcriptome of those plants capable of cold acclimation undergoes a complete reorganization, as revealed by the up- or downregulation of thousands of genes (Greenup et al., 2011; Laudencia-Chingcuanco et al., 2011). As estimated in Arabidopsis, more than 200 transcription factors are involved in the reconfiguration, and may serve as regulators for acclimation (Thomashow, 2010). The best understood cold regulatory pathway is the $\mathrm{CBF}$ regulon controlled by the C-repeat binding factors (CBFs), also called dehydration-responsive element binding (DREB1) factors (Thomashow, 2010; Mizoi et al., 2012). The CBFs belong to the AP2/EREBP (APETALA2/ethylene-responsive element binding protein) transcription factor family and possess a plant-specific AP2 DNA binding domain that interacts with the C-repeat elements present in the promoter region of their target genes (Jaglo et al., 2001). CBF expression is induced by different abiotic stresses (cold, drought, salt). The function of $C B F$ genes has been revealed in many plant species. In Arabidopsis, six $C B F$ s have been identified, while in the economically important cereals, the number of $C B F$ s are much higher: 20 in barley (Hordeum vulgare L.) (Skinner et al., 2005), 13 in einkorn (Triticum monococcum) (Miller et al., 2006) and 37 in common wheat (Triticum aestivum L.) (Badawi et al., 2007). CBF genes are positioned in clusters on the homeologous group 5 chromosomes of the Triticeae and coincide with the FR-2 quantitative trait locus (QTL) for freezing tolerance (Vágújfalvi et al., 2003, 2005; Miller et al., 2006; Tondelli et al., 2006; Båga et al., 2007; Francia et al., 2007). CBFs in Triticeae are regulated in a complex way, influenced by genotype, induction-temperature and lightregulated factors (Campoli et al., 2009). Analysis of 201 rye (Secale cereale L.) genotypes showed that single nucleotide polymorphisms (SNPs) in ScCBF15 and ScCBF12 genes were significantly associated with frost tolerance (Li et al., 2011). An einkorn mapping population was generated (Miller et al., 2006) and subjected to frost tests (Knox et al., 2008) and it was shown that three CBF genes (TmCBF12, TmCBF14 and TmCBF15) were responsible for the increased frost tolerance, and this improvement was related to higher expression levels of COR14b and DHN5 genes (Knox et al., 2008). In hexaploid wheat, three $C B F$ genes: TaCBF14, TaCBF15 and TaCBF16 were also induced by cold treatment; moreover, their enhanced expression was correlated with the level of frost tolerance (Vágújfalvi et al., 2005). In a comparative transcriptome analysis, six wheat $C B F \mathrm{~s}$ - among them $C B F 14-$ were found to be differentially expressed in the cold-acclimated winter wheat lines relative to the non-acclimated controls, thus suggesting a possible gain of function mutation that led to an increased level of frost tolerance. These mutations are considered as potential markers for frost survival (Sutton et al., 2009). The association analysis between genetic variants of $C B F s$ and freezing tolerance revealed that two nucleotide substitutions in $\mathrm{HvCBF} 14$ were statistically associated with freezing tolerance in a large European barley germplasm collection, suggesting that there was some degree of specificity among the different $C B F s$, and that $H v C B F 14$ was the most relevant one for frost tolerance (Fricano et al., 2009).

The role of the individual $C B F$ genes in temperate cereals is poorly understood, and their function has been tested by transformation methods in only a few experiments. Based on the above-mentioned results, TaCBF14 and TaCBF15 were isolated and overexpressed in spring barley to prove their function. The analysis showed that these 
transgenes indeed improved frost tolerance (Soltész et al., 2013). The expression of several target genes, regulated by the CBFs (HvCOR14b, HvDHN5 and HvDHN8), was enhanced in transgenic lines (Soltész et al., 2013). The overexpression of the barley $\mathrm{HvCBF} 4$ gene in transgenic rice resulted in increased tolerance to low temperature, drought and high salinity (Oh et al., 2007). Overexpression of the wheat CBF2 gene led to improved frost tolerance and enhanced expression of downstream genes in transgenic tobacco (Takumi et al., 2008). Constitutive overexpression of $H v C B F 2 A$ in spring barley led to greater freezing tolerance, as well as to enhanced transcript levels of HvCOR14b and HvDHN5 (Jeknič et al., 2014).

The experiments cited above show that members of the CBF transcription factor family are effective in improving freezing tolerance in diverse genetic backgrounds. In the future, the use of inducible or tissue-specific promoters may help to avoid the secondary effects (late flowering, retarded development) often found in transgenic plants overexpressing these genes.

\section{OsMYB4 transcription factors}

The MYB (myeloblastosis) transcription factor family is present in all eukaryotes, but plants encode a considerably larger number of $M Y B$ genes compared to fungi and animals (Katiyar et al., 2012). The MYB DNA-binding domain contains approximately 52 amino acid residues, and based on the number of adjacent MYB repeats, MYB transcription factors are classified into four major groups, namely 1R-MYB, 2R-MYB, 3R-MYB and 4R-MYB, containing one, two, three and four MYB repeats, respectively. The process of classification of MYBs is still in progress. Recently a genome-wide analysis led to the identification of 155 and 197 MYB genes in rice and Arabidopsis, respectively (Katiyar et al., 2012). The authors classified MYB transcription factors into four distinct groups namely 'MYB-related genes', 'MYB-R2R3', 'MYB-R1R2R3' and 'atypical $M Y B$ genes' based on the presence of one, two, three and four MYB repeats, respectively.
In plants, MYB transcription factors play a key role in plant development, hormone signal transduction, secondary metabolism, disease resistance and abiotic stress tolerance (Baldoni et al., 2013). From the different MYB classes, the R2R3-type OsMYB4 transcription factor of rice has been shown to play a special role in the regulation of several metabolic pathways during the acclimation to different abiotic stresses.

The function of OsMYB4 transcription factors was first revealed with the aid of Arabidopsis transgenic lines exposed to different stresses (cold, frost, drought, salt, ultraviolet (UV), ozone, viruses, bacteria and fungi). Not just improved tolerance/resistance to these conditions but also the activation of signal transduction processes involved in many different stress responses was shown in these transgenic lines (Vannini et al., 2004, 2006; Mattana et al., 2005). The importance of this gene was also demonstrated by the transformation of Arabidopsis and apple. The enhanced freezing and dehydration tolerance observed was considered a likely consequence of the accumulation of compatible osmolytes (Pasquali et al., 2008). The involvement of this transcription factor in the stress regulation process was also demonstrated indirectly, since several effector genes, such as COR15a, COR78 and D1-pyrroline5-carboxylate synthase (P5CS), also showed moderate induction in the Arabidopsis transgenic lines (Vannini et al., 2004, 2006; Mattana et al., 2005).

The expression of OsMYB4 transcript at three different levels in a Nipponbare rice genetic background shows that expression at a supraoptimal level leads to the misexpression of alternative targets with developmental side effects that affect panicle development (Park et al., 2010). Using transient expression assays, Baldoni et al. (2013) demonstrated that OsMyb4 was able to repress the activity of not only a paralogous gene, namely Os02g41510, but its own promoter, too. This 'feedback control' mechanism might explain the complexity of the transcript dynamics of the OsMyb4 transgenic plants, as well as the unexpected developmental alterations that were described by Park et al. (2010). 
The function of OsMyb4 was proved mainly in Arabidopsis. Until now, the effectiveness of this gene in cereals has been shown only in barley. Under the control of the stress-induced promoter COR15a, the overexpression of OsMYB4 gave improved cold tolerance in the spring barley Golden Promise. Transgenic lines had higher resistance to hypoxia and cold stress during germination. This work suggests a possible role of OsMYB4 in the development of flooding tolerance and in the facilitation of germination under unfavourable conditions (Soltész et al., 2012).

Considering the results described above, we agree with the conclusion drawn by Park et al. (2010): 'Transcription factors downstream to OsMYB4 appear to be the more ideal tools for regulon engineering because their individual effects are confined to specific defense mechanisms leading to positive net gains.'

\section{Changes During Prolonged Cold Acclimation}

Under prolonged cold conditions, the process of metabolic change continues, such as the accumulation of protective proteins, especially dehydrins, the downregulation of ABA levels and an elevation of positive regulators of cell division and growth (i.e. cytokinins, gibberellins and auxin) (Galiba et al., 2013; Vanková et al., 2014). These changes lead to the adaptation to low temperature, with a readjustment of metabolic activity to the less favourable conditions. According to recent views, the 'master' of these processes is the CBF regulon (Kurepin et al., 2013). Genes specific to this pathway encode, for example, other transcription factors, cold-regulated (Cor)/late embryogenesis abundant (Lea) genes, osmoprotectant biosynthesis proteins, carbohydrate metabolism-related proteins, sugar transport proteins, etc. (Vitámvás and Prásil, 2008; Zhou et al., 2011).

More importantly, apart from the regulation of the above-mentioned protective mechanisms, the CBF regulon affects plant development, especially by preventing growth and inducing a dwarf, compact phenotype, together with increased photosynthetic performance, typically associated with coldacclimated plants (reviewed by Kurepin et al., 2013). The CBF transcription factor was found to downregulate levels of active gibberellins by stimulating genes for the gibberellininactivating enzymes (GA 2-oxidases) and also by stabilizing DELLA proteins (named after the conserved Asp-Glu-Leu-Leu-Ala $\mathrm{N}$-terminal motif using their one-letter codes), which are repressors of the gibberellin signalling pathway (Achard et al., 2008; Soltész et al., 2013). The other important feature during cold acclimation is the maintenance of appropriate energy balance. Cold-hardy species, such as winter crops and Arabidopsis thaliana, exhibit an increase in photosynthetic capacity through the upregulation of carbon metabolism during cold acclimation (Hurry et al., 1995; Hüner et al., 1998; Stitt and Hurry, 2002; Dahal et al., 2012). This process leads to global reprogramming of photosynthetic carbon metabolism (Gray and Heath, 2005). The cold acclimationinduced stimulation in photosynthetic capacity is correlated positively with the development of freezing tolerance, as well as with an increased resistance to low temperature-induced photoinhibition in winter rye and winter wheat (Gray et al., 1996; Pocock et al., 2001). Most likely, the photosynthetic carbon metabolism-related acclimation process is also linked, at least partly, to the CBF regulon. It was shown recently that the expression of AtCBF3 appeared to be governed by chloroplast excitation pressure modulated by either low temperature or high light (Bode, 2013). Moreover, overexpression of CBFs in different plant species mimics the cold acclimation process, and the enhanced photosynthetic performance associated with cold acclimation (Savitch et al., 2005; Yang et al., 2010; Dahal et al., 2012; Lee and Thomashow, 2012). Accordingly, we describe below the most important metabolites where accumulation during the cold acclimation process is characteristic, and which are overwhelmingly used as markers to prove the enhanced freezing tolerance of the genetically modified plants. 


\section{Carbohydrates}

As described above, there is a positive correlation between photosynthetic performance and cold acclimation. Consequently, there must also be some correlation between the carbohydrate content of the different plant tissues and the manifestation of the actual freezing tolerance. Indeed, early studies on spring and overwintering crops have shown that freezing tolerance is correlated strongly with the capacity to increase soluble carbohydrate pools during cold hardening (Tognetti et al., 1990; Öquist et al., 1993; Hurry et al., 1995). Furthermore, field studies have shown that plants become vulnerable to freezing injury when the fructan pool becomes depleted and simple sugars can no longer be released into the cytosol and intracellular liquid (Olien and Clark, 1993). Association between freezing tolerance and carbohydrate accumulation was also demonstrated using genetic tools. QTLs controlling traits associated with winter hardiness in barley, including field survival, LT50 (temperature lethal to $50 \%$ of a test population), growth habit and crown fructan content, were mapped to chromosome 7 (Hayes et al., 1993). The positive correlation between carbohydrate accumulation and freezing tolerance was also revealed in experiments using chromosome substitution analysis in wheat (Galiba et al., 1997; Vágújfalvi et al., 1999). It was shown that 5A and 5D chromosomes not only increased freezing tolerance but also increased the accumulation of carbohydrates in a time-dependent manner (Vagújfalvi et al., 1999). These results are in agreement with the recent view, summarized below, regarding the role of fructans in freezing tolerance (see details in recent excellent reviews by Livingston et al., 2009, and Yoshida and Kawakami, 2013).

Fructans are water-soluble carbohydrates that accumulate in vacuoles and act as storage carbohydrates in a large number of plant species. Wheat also uses fructan as a temporal photoassimilate instead of starch in plastids. Temperate grasses accumulate a levan type of fructan, which is composed primarily of $\beta(2 \rightarrow 1)$ - and $\beta(2 \rightarrow 1)$-linked fructosyl units, which are biosynthesized by sucrose:sucrose 1-fructosyltransferase (1-SST wft2, AB029888), sucrose:fructan 6-fructosyltransferase (6-SFT wft1, AB029887; Kawakami and Yoshida, 2002) and fructan:fructan 1-fructosyltransferase enzymes, while it is degraded by fructan exohydrolase (FEH) (Gallagher et al., 2007; Yoshida and Tamura, 2011).

Transgenic approaches proved to be very useful in verifying the role of key enzymes in fructan metabolism, and also in monitoring the changes in physiology-related traits. Transgenic perennial ryegrass (Lolium perenne) that overexpressed wheat 1-SST and 6 -SFT genes under the control of the constitutive CaMV $35 S$ promoter accumulated an increased level of fructan. These transgenic plants also had increased tolerance to freezing at the cellular level (Hisano et al., 2004). Transgenic perennial ryegrass overexpressing onion 1-SST and 6G-FFT (fructan:fructan 6G-fructosyltransferase) genes showed increased fructan levels up to threefold higher in leaf and stem tissues compared to the wild type (Gadegaard et al., 2008). Rice plants overexpressing wheat 6-SFT and 1-SST genes accumulated more fructans in the leaves and in the stems, and 1-SST transgenic rice seedlings exhibited enhanced chilling tolerance as well (Kawakami et al., 2008). In a study where rye 1 -SST and wheat 6 -SFT genes were overexpressed under the control of a wheat aleurone layer-specific promoter (ns-LTP) in triticale plants, it was shown that the transgenic lines accumulated some 50\% less starch and 10-20 times more fructan in the dry seed compared to the wild type. These fructans were metabolized rapidly during germination, and in the transgenic lines, where both the 1-SST and 6-SFT genes were overexpressed, the fructan contents were significantly higher compared to the wild type. Also, during low-temperature germination, an increased synthesis of fructans was observed in the transgenics (Diedhiou et al., 2012).

\section{Proline}

Apart from the dynamic changes of carbohydrate content, significant changes in the content of hundreds of other metabolites 
have been reported during both cold and sub-zero acclimation (Pearce, 2004; Zhu et al., 2007; Henson et al., 2014). We will consider here only the free amino acids, although other metabolites, like polyamines, nitric oxide and glutathione, are essential in redox control during cold acclimation (Galiba et al., 2013; Kocsy et al., 2013).

Most of the metabolite-related studies during cold acclimation were carried out on the model plant Arabidopsis (Cook et al., 2004; Kaplan et al., 2004; Guy et al., 2008). The importance of the free amino acids, especially proline, in abiotic stress tolerance of plants has been well established for a long time. The amino acids have several roles in plants; for example, they act as osmolytes, detoxify heavy metals, regulate ion transport and stomatal opening, and affect the synthesis and activity of enzymes, and influence gene expression and redox homeostasis (Rai, 2002). Proline, as a source of osmolite, could have a special role during cold acclimation, since its accumulation may prevent water loss occurring from cells at sub-zero temperatures, due to extracellular ice formation. Indeed, the proline content increased in a variety of plant species (orange, potato, wheat, etc.) during cold acclimation. Moreover, supplying proline to potato exogenously increased its freezing tolerance (Yelenosky, 1979; van Swaaij et al., 1985; Dörffling et al., 1990; Macháčcková et al., 2006).

High proline content has been considered as a marker for drought tolerance in cereal breeding programmes, and even in winter barley the elevated proline levels in cold-hardened leaves was used as an early selection criterion for freezing-tolerant lines (Winkel, 1989). However, we now realize that this was a rather simplified assumption. First of all, in plants, there is a large overlap between cold-regulated and circadian-regulated genes (Fowler, 2008). Carrying out metabolic profiling, Espinoza et al. (2010) found about $80 \%$ of metabolites that showed diurnal cycles maintained these oscillations during cold treatment. So, the level of the metabolites is fluctuating even on a daily basis, which makes the interpretation of the actual concentration dubious. Second, in a recent study, where 54 Arabidopsis accessions with diverse geographical origin were compared, it was concluded that leaf glucose, fructose, sucrose and raffinose contents were correlated with freezing tolerance in the cold-acclimated state, while proline content was not (Zuther et al., 2012). However, in a Thellungiella germplasm collection, the proline content was in correlation with freezing tolerance (Lee et al., 2012). Although cold treatment triggered the accumulation of proline in Brachypodium, the size of the proline pool of a given accession could not be used to predict its freezing tolerance behaviour accurately (Colton-Gagnon et al., 2014). Comparing three wheat cultivars with different freezing tolerances, the proline content was found to have increased in all of the cultivars after one week of cold acclimation, but a prolonged cold acclimation resulted in different profiles: no further increase occurred in the most sensitive cultivar, while an additional increase occurred in the other two cultivars (Kamata and Uemura, 2004). It was reported that accumulation of proline at low temperature in wheat was accompanied by an elevated level of ABA, a hormone involved in stress signalling (Macháčcková et al., 2006; Tuteja, 2007). In a study using CS/Cheyenne disomic chromosome substitution lines, the substituted 5A chromosome from the winter hardy Cheyenne cultivar increased both the freezing tolerance and the ABA content in the recipient, cold-sensitive Chinese Spring (CS) genetic background (Galiba et al., 1993). So, an open question appeared: what is the relationship between the ABA and free amino acid content during the cold-acclimation process? To get the answer, the effect of cold acclimation and ABA on amino acid content was compared, using the same disomic chromosome substitution lines mentioned above (Kovács et al., 2011). Cold acclimation induced the accumulation of most of the amino acids; while ABA had a significant effect only on asparagine. So, the cold-induced changes in free amino acid levels were probably not mediated by ABA. Chromosome 5A may affect the cold-induced free amino acid content, through the activation of the $C B F$ gene cluster, present at the 
Fr-A2 locus in Triticeae (Atienza et al., 2004). This hypothesis is supported by results published on Arabidopsis, as described below (Cook et al., 2004).

To clarify the rather cloudy picture on the possible involvement of proline in abiotic stress tolerance, several proline-related transgenic studies were conducted (Szabados and Savouré, 2010). Transgenic Arabidopsis plants with an antisense AtProDH cDNA encoding proline dehydrogenase (which catalyses proline degradation) were generated (Nanjo et al., 1999). Several transgenics showed enhanced accumulation of proline, and these transgenic plants also showed tolerance to freezing and high salinity. In another experiment, it was demonstrated that there was around an $80 \%$ overlap between the metabolic responses of the constitutive overexpressing $C B F 3$ non-acclimated transgenic and non-transgenic cold-acclimated plants, indicating that the changes in the alteration of many metabolites (including proline) were regulated by the $C B F$ genes in Arabidopsis (Cook et al., 2004). The connection between $C B F$ genes and proline metabolism was confirmed more recently in a transgenic maize system. The overexpression of TsCBF1 from Thellungiella halophila in maize plants subjected to drought stress resulted in increased pyrroline-5-carboxylate synthetase gene transcription, elevated proline content, and also in higher stress tolerance (Zhang et al., 2010). So, we could conclude that it is likely the CBF regulon is responsible for the metabolic changes during cold acclimation.

Some other experiments, using transgenic plants, might also shed light on the puzzle of why the relation between proline accumulation and abiotic stress tolerance (especially for frost tolerance) is not really tight in each and every case. The Vigna aconitifolia D1-pyrroline-5-carboxylate synthetase (P5CS) gene that encodes the key regulatory enzyme in proline biosynthesis has been transformed into wheat, and increased tolerance to water deficit has been shown in the transgenic lines (Vendruscolo et al., 2007). However, the authors concluded that the gained tolerance to water deficit observed in transgenic plants was not due to increased osmotic adjustment, but instead was due mainly to the induced protection mechanisms against oxidative stresses. It has also been reported that the higher proline accumulation in P5CS-transformed tobacco plants reduced free radical levels, measured by MDA (malondialdehyde) content, in response to osmotic stress (Parvanova et al., 2004).

From the results described above, it is clear that proline can be considered as a multifunctional amino acid, interacting with various metabolic pathways - especially with antioxidants - to ameliorate the constraints of abiotic stresses. That is why its accumulation rate cannot be considered in the same way as a 'single trait' of stress tolerance. However, even considering this fact, we can still support the conclusion of Szabados and Savouré (2009), who stated that the engineering of proline metabolism could lead to new opportunities to improve plant tolerance of environmental stresses.

\section{Conclusions}

Molecular biology advances, in parallel with improved transformation methodology, have opened up a great opportunity to develop more stress-tolerant cereal varieties, in addition to facilitating our understanding of the function of the genes involved. In spite of the fact that, nowadays, experiments with transgenic plants are restricted mainly to testing and proving target gene function, in the (near) future these experiments will result in plant materials ready to be provided to breeders to allow the development of commercial varieties.

\section{Acknowledgements}

The authors are thankful for the support of the Hungarian Research Fund 'OTKA' No. K75528, K80781, K11879, EU BONUS 12-12012-0024 and ADAPTAWHEAT project FP7KBBE-2011-5 (Project No. 289842). Alexandra Soltész is a recipient of the János Bolyai Research Grant. 


\section{References}

Achard, P., Gong, F., Cheminant, S., Alioua, M., Hedden, P. and Genschik, P. (2008) The cold-inducible CBF1 factor-dependent signaling pathway modulates the accumulation of the growth-repressing DELLA proteins via its effect on gibberellin metabolism. Plant Cell 20, 2117-2129.

Atienza, S.G., Faccioli, P., Perrotta, G., Dalfino, G., Zschiesche, W., et al. (2004) Large scale analysis of transcripts abundance in barley subjected to several single and combined abiotic stress conditions. Plant Science 167, 1359-1365.

Badawi, M., Danyluk, J., Boucho, B., Houde, M. and Sarhan, F. (2007) The CBF gene family in hexaploid wheat and its relationship to the phylogenetic complexity of cereal CBFs. Molecular Genetics and Genomics 277, 533-554.

Båga, M., Chodaparambil, S.V., Limin, A.E., Pecar, M., Fowler, D.B. and Chibbar, R.N. (2007) Identification of quantitative trait loci and associated candidate genes for low-temperature tolerance in cold-hardy winter wheat. Functional and Integrative Genomics 7, 53-68.

Baldoni, E., Genga, A., Medici, A., Coraggio, I. and Locatelli, F. (2013) The OsMyb4 gene family: stress response and transcriptional auto-regulation mechanisms. Biologia Plantarum 57, 691-700.

Bode, R. (2013) Effects of excitation pressure on variegation and global gene expression in Arabidopsis thaliana. PhD thesis. Western University, Canada.

Campoli, C., Matus-Cadiz, M.A., Pozniak, C.J., Cattivelli, L. and Fowler, D.B. (2009) Comparative expression of $C B F$ genes in the Triticeae under different acclimation induction temperatures. Molecular Genetics and Genomics 282, 141-152.

Colton-Gagnon, K., Ali-Benali, M.A., Mayer, B.F., Dionne, R., Bertrand, A., et al. (2014) Comparative analysis of the cold acclimation and freezing tolerance capacities of seven diploid Brachypodium distachyon accessions. Annals of Botany 113, 681-693.

Cook, D., Fowler, S., Fiehn, O. and Thomashow, M.F. (2004) A prominent role for the CBF cold response pathway in configuring the low temperature metabolome of Arabidopsis. Proceedings of the National Academy of Sciences of the United States of America 101, 15243-15248.

Dahal, K., Kane, K., Gadapati, W., Webb, E., Savitch, L.V., et al. (2012) The effects of phenotypic plasticity on photosynthetic performance in winter rye, winter wheat and Brassica napus. Physiologia Plantarum 144, 169-188.

Diedhiou, C., Gaudet, D., Liang, Y., Sun, J., Lu, Z.X., et al. (2012) Carbohydrate profiling in seeds and seedlings of transgenic triticale modified in the expression of sucrose:sucrose-1-fructosyltransferase (1-SST) and sucrose:fructan-6-fructosyltransferase (6-SFT). Journal of Bioscience and Bioengineering 114, 371-378.

Dörffling, K., Schulenburg, S., Lesselich, G. and Dörffling, H. (1990) Abscisic acid and proline levels in cold-hardened winter wheat leaves in relation to variety-specific differences in freezing resistance. Journal of Agronomy and Crop Science 165, 230-239.

Dunwell, J.M. (2014) Transgenic cereals: current status and future prospects. Journal of Cereal Science 59, 419-434.

Espinoza, C., Degenkolbe, T., Caldana, C., Zuther, E., Leisse, A., et al. (2010) Interaction with diurnal and circadian regulation results in dynamic metabolic and transcriptional changes during cold acclimation in Arabidopsis. PLoS ONE 5, e14101.

Fowler, D.B. (2008) Cold acclimation threshold induction temperatures in cereals. Crop Science 48, 1147-1154.

Francia, E., Barabaschi, D., Tondelli, A., Laido, G., Rizza, F., et al. (2007) Fine mapping of a HvCBF gene cluster at the frost resistance locus Fr- H2 in barley. Theoretical and Applied Genetics 115, 1083-1091.

Franklin, K.A. (2009) Light and temperature signal crosstalk in plant development. Current Opinion in Plant Biology 12, 63-68.

Franklin, K.A. and Whitelam, G.C. (2007) Light-quality regulation of freezing tolerance in Arabidopsis thaliana. Nature Genetics 39, 1410-1413.

Fricano, A., Rizza, F., Faccioli, P., Pagani, D., Pavan, P., et al. (2009) Genetic variants of HvCBF14 are statistically associated with frost tolerance in a European germplasm collection of Hordeum vulgare. Theoretical and Applied Genetics 119, 1335-1348.

Gadegaard, G., Didion, T., Folling, M., Storgaard, M., Andersen, C.H. and Nielsen, K.K. (2008) Improved fructan accumulation in perennial ryegrass transformed with the onion fructosyltransferase genes 1-SST and 6G-FFT. Journal of Plant Physiology 165, 1214-1225.

Galiba, G., Tuberosa, R., Kocsy, G. and Sutka, J. (1993) Involvement of chromosome 5A and 5D in coldinduced abscisic acid accumulation and in frost tolerance of wheat calli. Plant Breeding 110, 237-242. 
Galiba, G., Kerepesi, I., Snape, J.W. and Sutka, J. (1997) Location of a gene regulating cold-induced carbohydrate production on chromosome 5A of wheat. Theoretical and Applied Genetics 95, 265-270.

Galiba, G., Vankova, R., Tari, M., Bánfalvi, Zs., Poór, P., et al. (2013) Hormones, NO, antioxidants and metabolites as key players in plant cold acclimation. In: Imai, R., Yoshida, M. and Matsumoto, N. (eds) Plant and Microbe Adaptations to Cold in a Changing World. Springer Science+Business Media, New York, pp. 73-88.

Gallagher, J.A., Cairns, A.J. and Turner, L.B. (2007) Fructan in temperate forage grasses: agronomy, physiology. In: Shiomi, N., Benkeblia, N. and Onodera, S. (eds) Recent Advances in Fructooligosaccharides Research. Research Signpost Publisher, Kerala, India, pp. 15-46.

Gray, G.R. and Heath, D. (2005) A global reorganization of the metabolome in Arabidopsis during cold acclimation is revealed by metabolic fingerprinting. Physiologia Plantarum 124, 236-248.

Gray, G.R., Savitch, L.V., Ivanov, A.G. and Hüner, N.P.A. (1996) Photosystem II excitation pressure and development of resistance to photoinhibition: II. Adjustment of photosynthetic capacity in winter wheat and winter rye. Plant Physiology 110, 61-71.

Greenup, A.G., Sasani, S., Oliver, S.N., Walford, S.A., Millar, A.A. and Trevaskis, B. (2011) Transcriptome analysis of the vernalization response in barley (Hordeum vulgare) seedlings. PLoS ONE 6(3), e17900.

Guy, C., Kaplan, F., Kopka, J., Selbig, J. and Hincha, D.K. (2008) Metabolomics of temperature stress. Physiologia Plantarum 132, 220-235.

Hayes, P.M., Blake, T.K., Chen, T.H.H., Tragoonrung, S., Chen, F., et al. (1993) Quantitative trait loci on barley (Hordeum vulgare) chromosome 7 associated with components of winter hardiness. Genome 36, 66-71.

Henson, C.A., Duke, S.H. and Livingston, D.P. III (2014) Metabolic changes in Avena sativa crowns recovering from freezing. PLOS ONE 9(3), e93085.

Hisano, H., Kanazawa, A., Kawakami, A., Yoshida, M., Shimamoto, Y. and Yamada, T. (2004) Transgenic perennial ryegrass plants expressing wheat fructosyltransferase genes accumulate increased amounts of fructan and acquire increased tolerance on a cellular level to freezing. Plant Science 167, 861-868.

Hüner, N.P.A., Öquist, G. and Sarhan, F. (1998) Energy balance and acclimation to light and cold. Trends in Plant Science 3, 224-230.

Hurry, V.M., Strand, A., Tobiaeson, M., Gardeström, P. and Öquist, G. (1995) Cold hardening of spring and winter wheat and rape results in differential effects on growth, carbon metabolism, and carbohydrate content. Plant Physiology 109, 697-706.

Jaglo, K.R., Kleff, S., Amundsen, K.L., Zhang, X., Haake, V., et al. (2001) Components of the Arabidopsis C-repeat/ dehydration-responsive element binding factor cold response pathway are conserved in Brassica napus and other plant species. Plant Physiology 127, 910-917.

Jeknič, Z., Pillman, K.A., Dhillon, T., Skinner, J.S., Veisz, O., et al. (2014) Hv-CBF2A overexpression in barley accelerates $C O R$ gene transcript accumulation and acquisition of freezing tolerance during cold acclimation. Plant Molecular Biology 84, 67-82.

Kamata, T. and Uemura, M. (2004) Solute accumulation in wheat seedlings during cold acclimation: contribution to increased freezing tolerance. CryoLetters 25, 311-322.

Kaplan, F., Kopka, J., Haskell, D.W., Zhao, W., Schiller, K.C., et al. (2004) Exploring the temperature-stress metabolome of Arabidopsis. Plant Physiology 136, 4159-4168.

Katiyar, A., Smita, S., Lenka, S.K., Rajwanshi, R., Chinnusamy, V. and Bansal, K.C. (2012) Genome-wide classification and expression analysis of $M Y B$ transcription factor families in rice and Arabidopsis. BMC Genomics 13, 544.

Kawakami, A. and Yoshida, M. (2002) Molecular characterization of sucrose:sucrose 1-fructosyltransferase and sucrose:fructan 6-fructosyltransferase associated with fructan accumulation in winter wheat during cold hardening. Bioscience, Biotechnology, and Biochemistry 66, 2297-2305.

Kawakami, A., Sato, Y. and Yoshida, M. (2008) Genetic engineering of rice capable of synthesizing fructans and enhancing chilling tolerance. Journal of Experimental Botany 59, 793-802.

Knox, A.K., Li, C., Vágújfalvi, A., Galiba, G., Stockinger, E.J. and Dubcovsky, J. (2008) Identification of candidate $C B F$ genes for the frost tolerance locus $F r-A^{m} 2$ in Triticum monococcum. Plant Molecular Biology $67,257-270$.

Kocsy, G., Tari, I., Vanková, R., Zechmann, B., Gulyás, Zs., et al. (2013) Redox control of plant growth and development. Plant Science 211, 77-91.

Kosová, K., Prasil, I.T., Vitamvas, P., Dobrev, P., Motyka, V., et al. (2012) Complex phytohormone responses during the cold acclimation of two wheat cultivars differing in cold tolerance, winter Samanta and spring Sandra. Journal of Plant Physiology 169, 567-576. 
Kovács, Z., Simon-Sarkadi, L., Sovány, C., Kirsch, K., Galiba, G. and Kocsy, G. (2011) Differential effects of cold acclimation and abscisic acid on free amino acid composition in wheat. Plant Science 180, 61-68.

Kurepin, L.V., Dahal, K.P., Savitch, L.V., Singh, J., Bode, R., et al. (2013) Role of CBFs as integrators of chloroplast redox, phytochrome and plant hormone signaling during cold acclimation. International Journal of Molecular Sciences 14, 12729-12763.

Laudencia-Chingcuanco, D., Ganeshan, S., You, F., Fowler, B., Chibbar, R. and Anderson, O. (2011) Genome-wide gene expression analysis supports a developmental model of low temperature tolerance gene regulation in wheat (Triticum aestivum L.). BMC Genomics 12, 299.

Lee, C.M. and Thomashow, M.F. (2012) Photoperiodic regulation of the C-repeat binding factor (CBF) cold acclimation pathway and freezing tolerance in Arabidopsis thaliana. Proceedings of the National Academy of Sciences of the United States of America 109, 15054-15059.

Lee, Y.P., Babakov, A., de Boer, B., Zuther, E. and Hincha, D.K. (2012) Comparison of freezing tolerance, compatible solutes and polyamines in geographically diverse collections of Thellungiella sp. and Arabidopsis thaliana accessions. BMC Plant Biology 12, 131.

Li, Y.L., Bock, A., Haseneyer, G., Korzun, V., Wilde, P., et al. (2011) Association analysis of frost tolerance in rye using candidate genes and phenotypic data from controlled, semi-controlled, and field phenotyping platforms. BMC Plant Biology 11, 146-160.

Livingston, D.P., Hincha, D.K. and Heyer, A.G. (2009) Fructan and its relationship to abiotic stress tolerance in plants. Cellular and Molecular Life Sciences 66, 2007-2023.

Macháčcková, I., Hanišová, A. and Krekule, J. (2006) Levels of ethylene, ACC, MACC, ABA and proline as indicators of cold hardening and frost resistance in winter wheat. Physiologia Plantarum 76, 603-607.

Mattana, M., Biazzi, E., Consonni, R., Locatelli, F., Vannini, C., et al. (2005) Overexpression of Osmyb4 enhances compatible solute accumulation and increases stress tolerance of Arabidopsis thaliana. Physiologia Plantarum 125, 212-223.

Miller, A.K., Galiba, G. and Dubcovsky, J. (2006) A cluster of 11 CBF transcription factors is located at the frost tolerance locus Fr-A $\mathrm{A}^{m} 2$ in Triticum monococcum. Molecular Genetics and Genomics 275, 193-203.

Mizoi, J., Shinozaki, K. and Yamaguchi-Shinozaki, K. (2012) AP2/ ERF family transcription factors in plant abiotic stress responses. Biochimica et Biophysica Acta 1819, 86-96.

Nanjo, T., Kobayashi, M., Yoshiba, Y., Kakubari, Y., Yamaguchi-Shinozaki, K. and Shinozaki, K. (1999) Antisense suppression of proline degradation improves tolerance to freezing and salinity in Arabidopsis thaliana. FEBS Letters 461, 205-210.

Oh, S.J., Kwon, C.W., Choi, D.W., Song, S.I. and Kim, J.K. (2007) Expression of barley HvCBF4 enhances tolerance to abiotic stress in transgenic rice. Plant Biotechnology Journal 5, 646-656.

Olien, C.R. and Clark, J.L. (1993) Changes in soluble carbohydrate composition of barley, wheat, and rye during winter. Agronomy Journal 85, 21-29.

Öquist, G., Hurry, V.M. and Hüner, N.P.A. (1993) Low temperature effects on photosynthesis and correlation with freezing tolerance in spring and winter cultivars of wheat and rye. Plant Physiology 101, 245-250.

Park, M.R., Yun, K.Y., Mohanty, B., Herath, V., Xu, F., et al. (2010) Supra-optimal expression of the coldregulated OsMyb4 transcription factor in transgenic rice changes the complexity of transcriptional network with major effects on stress tolerance and panicle development. Plant, Cell and Environment 33, 2209-2230.

Parvanova, D., Ivanov, S., Konstantinova, T., Karanov, E., Atanassov, A., et al. (2004) Transgenic tobacco plants accumulating osmolytes show reduced oxidative damage under freezing stress. Plant Physiology and Biochemistry 42, 57-63.

Pasquali, G., Biricolti, S., Locatelli, F., Baldoni, E. and Mattana, M. (2008) Osmyb4 expression improves adaptive responses to drought and cold stress in transgenic apples. Plant Cell Reports 27, 1677-1686.

Pearce, R.S. (2004) Adaptation of higher plants to freezing. In: Fuller, B.J., Lane, N., Benson, E.E. (eds) Life in the Frozen State. CRC Press LLC, Boca Raton, Florida, pp. 171-204.

Pocock, T.H., Hurry, V., Savitch, L.V. and Hüner, N.P.A. (2001) Susceptibility to low-temperature photoinhibition and the acquisition of freezing tolerance in winter and spring wheat: the role of growth temperature and irradiance. Physiologia Plantarum 113, 499-506.

Rai, V.K. (2002) Role of amino acids in plant responses to stresses. Biologia Plantarum 45, 481-487.

Sandre, S.L., Tammaru, T. and Hokkanen, H.M. (2011) Pathogen resistance in the moth Orgyia antiqua: direct influence of host plant dominates over the effects of individual condition. Bulletin of Entomological Research 101, 107-114.

Sanghera, G.S., Wani, S.H., Hussain, W. and Singh, N.B. (2011) Engineering cold stress tolerance in crop plants. Current Genomics 12, 30-43. 
Savitch, L.V., Allard, G., Seki, M., Robert, L.S., Tinker, N.A., et al. (2005) The effect of overexpression of two Brassica CBF/DREB1-like transcription factors on photosynthetic capacity and freezing tolerance in Brassica napus. Plant and Cell Physiology 46, 1525-1539.

Skinner, J.S., von Zitzewitz, J., Szúcs, P., Marquez-Cedillo, L., Filichkin, T., et al. (2005) Structural, functional, and phylogenetic characterization of a large CBF gene family in barley. Plant Molecular Biology 59, 533-551.

Soltész, A., Vágújfalvi, A., Rizza, F., Kerepesi, I., Galiba, G., et al. (2012) The rice OsMYB4 gene enhances tolerance to frost and improves germination under unfavourable conditions in transgenic barley plants. Journal of Applied Genetics 53, 133-143.

Soltész, A., Smedley, M., Vashegyi, I., Galiba, G., Harwood, W. and Vágújfalvi, A. (2013) Transgenic barley lines prove the involvement of TaCBF14 and TaCBF15 in the cold acclimation process and in frost tolerance. Journal of Experimental Botany 64, 1849-1862.

Stitt, M. and Hurry, V. (2002) A plant for all seasons: alterations in photosynthetic carbon metabolism during cold acclimation in Arabidopsis. Current Opinion in Plant Biology 5, 199-206.

Sutton, F., Chen, D.G., Ge, X. and Kenefick, D. (2009) CBF genes of the Fr-A2 allele are differentially regulated between longterm cold acclimated crown tissue of freeze-resistant and -susceptible, winter wheat mutant lines. BMC Plant Biology 9, 34-42.

Swaaij, A.C. van, Jacobsen, E. and Feenstra, W.J. (1985) Effect of cold hardening, wilting and exogenously applied proline on leaf proline content and frost tolerance of several genotypes of Solanum. Physiologia Plantarum 64, 230-236.

Szabados, L. and Savouré, A. (2010) Proline: a multifunctional amino acid. Trends in Plant Science 15, 89-97.

Takumi, S., Shimamura, C. and Kobayashi, F. (2008) Increased freezing tolerance through up-regulation of downstream genes via the wheat CBF gene in transgenic tobacco. Plant Physiology and Biochemistry 46, 205-211.

Thomashow, M.F. (1999) Plant cold acclimation: freezing tolerance genes and regulatory mechanisms. Annual Review of Plant Physiology and Plant Molecular Biology 50, 571-599.

Thomashow, M.F. (2010) Molecular basis of plant cold acclimation: insights gained from studying the CBF cold responsive pathway. Plant Physiology 154, 571-577.

Tognetti, J.A., Salerno, G.L., Crespi, M.D. and Pontis, H.G. (1990) Sucrose and fructan metabolism of different wheat cultivars at chilling temperatures. Physiologia Plantarum 78, 554-559.

Tondelli, A., Francia, E., Barabaschi, D., Aprile, A., Skinner, J.S., et al. (2006) Mapping regulatory genes as candidates for cold and drought stress tolerance in barley. Theoretical and Applied Genetics 112, 445-454.

Tuteja, N. (2007) Abscisic acid and abiotic stress signaling. Plant Signaling and Behavior 2, 135-138.

Vágújfalvi, A., Kerepesi, I., Galiba, G., Tischner, T. and Sutka, J. (1999) Frost hardiness depending on carbohydrate changes during cold acclimation in wheat. Plant Science 144, 85-92.

Vágújfalvi, A., Galiba, G., Cattivelli, L. and Dubcovsky, J. (2003) The cold regulated transcriptional activator CBF3 is linked to the frost-tolerance locus Fr-A2 on wheat chromosome 5A. Molecular Genetics and Genomics 269, 60-67.

Vágújfalvi, A., Aprile, A., Miller, A., Dubcovsky, J., Delugu, J., et al. (2005) The expression of several CBF genes at the Fr-A2 locus is linked to frost tolerance in wheat. Molecular Genetics and Genomics 274, 506-514.

Vanková, R., Kosová, K., Dobreva, P., Vítámvás, P., Trávnícková, A., et al. (2014) Dynamics of cold acclimation and complex phytohormone responses in Triticum monococcum lines G3116 and DV92 differing in vernalization and frost tolerance level. Environmental and Experimental Botany 101, 12-25.

Vannini, C., Locatelli, F., Bracale, M., Magnani, E., Marsoni, M., et al. (2004) Overexpression of the rice Osmyb4 gene increases chilling and freezing tolerance of Arabidopsis thaliana plants. The Plant Journal $37,115-127$.

Vannini, C., Iriti, M., Bracale, M., Locatelli, F., Faoro, F., et al. (2006) The ectopic expression of the rice Osmyb4 gene in Arabidopsis increases tolerance to abiotic, environmental and biotic stresses. Physiological and Molecular Plant Pathology 69, 26-42.

Vendruscolo, E.C., Schuster, I., Pileggi, M., Scapim, C.A., Molinari, H.B., et al. (2007) Stress-induced synthesis of proline confers tolerance to water deficit in transgenic wheat. Journal of Plant Physiology 164, 1367-1376.

Vítámvás, P. and Prásil, I.T. (2008) WCS120 protein family and frost tolerance during cold acclimation, deacclimation and reacclimation of winter wheat. Plant Physiology and Biochemistry 46, 970-976.

Winkel, A. (1989) Breeding for drought tolerance in cereals. Vorträge Pflanzenzücht 16, 357-368. 
Yang, J.S., Wang, R., Meng, J.J., Bi, Y.P., Xu, P.L., et al. (2010) Overexpression of Arabidopsis CBF1 gene in transgenic tobacco alleviates photoinhibition of PSII and PSI during chilling stress under low irradiance. Journal of Plant Physiology 167, 534-539.

Yelenosky, G. (1979) Accumulation of free proline in citrus leaves during cold hardening of young trees in controlled temperature regimes. Plant Physiology 64, 425-427.

Yoshida, M. and Kawakami, A. (2013) Molecular analysis of fructan metabolism associated with freezing tolerance and snow mold resistance of winter wheat. In: Imai, R., Yoshida, M. and Matsumoto, N. (eds) Plant and Microbe Adaptations to Cold in a Changing World. Springer Science+Business Media, New York, pp. 231-243.

Yoshida, M. and Tamura, K. (2011) Research on fructan in wheat and temperate grasses in Japan. Japan Agricultural Research Quarterly 45, 9-14.

Zhang, S., Li, N., Gao, F., Yang, A. and Zhang, J. (2010) Over-expression of TSCBF1 gene confers improved drought tolerance in transgenic maize. Molecular Breeding 26, 455-465.

Zhou, M.Q., Shen, C., Wu, L.H., Tang, K.X. and Lin, J. (2011) CBF-dependent signaling pathway: a key responder to low temperature stress in plants. Critical Reviews in Biotechnology 31, 186-192.

Zhu, J., Dong, C-H. and Zhu, J-K. (2007) Interplay between cold-responsive gene regulation, metabolism and RNA processing during plant cold acclimation. Current Opinion in Plant Biology 10, 290-295.

Zuther, E., Schulz, E., Childs, L.H. and Hincha, D.K. (2012) Clinal variation in the nonacclimated and cold acclimated freezing tolerance of Arabidopsis thaliana accessions. Plant, Cell and Environment 35, 1860-1878. 